\title{
Parallel processing of stimulus features during RSVP: Evidence from the second response
}

\author{
Juan Botella, María Narváez, and Manuel Suero \\ Autónoma University of Madrid, Madrid, Spain \\ AND \\ JAMES F. JUOLA \\ University of Kansas, Lawrence, Kansas
}

\begin{abstract}
When observers are asked to report a feature of a single target displayed in rapid serial visual presentation, they frequently make errors. Most frequently, a feature from the to-be-reported dimension pertaining to a stimulus presented near the target is reported. These migrations are so-called illusory conjunctions in the time domain. From parallel models, it is proposed that during the presentation of the series, the response features of the stimuli are extracted. If a high rate of presentation does not enable proper binding processes, the system could base its response on sophisticated guessing on the basis of the relative levels of activation of the available response features. However, the multiple extractions assumption has not received direct empirical support. We report two experiments in which the observers had to report their first and second response candidates. This double response paradigm makes it possible to test the assumption that more than one response feature is available for making a response. Furthermore, the application of the constant ratio rule (following Botella, Barriopedro, \& Suero's, 2001, model) to the first responses allows predictions for the ratios between choices of the items for the second responses. The correlations between the observed and the predicted response proportions were .887 and .956 in the two experiments. This high predictive capacity indicates, first, that the observers have more than one response available, among which to choose, and second, that the choice among responses is determined largely in the same way for both first and second responses. Nevertheless, the small errors in prediction are further reduced if a proportion of pure guesses is assumed in the second responses. These are probably due to memory losses, misidentifications of the features, and other factors impairing performance in second responses in comparison with first responses.
\end{abstract}

The last two decades have witnessed a remarkable increase in interest in human temporal capacity limitations in processing environmental stimuli, and effects on performance when these limits are exceeded (Shapiro, 2001). One manifestation of such limits, as viewed from the conceptual framework of attention, has been the difficulty in noticing stimulus repetitions if the two occurrences are close in time (repetition blindness or RB; Kanwisher, 1991). Another manifestation is the difficulty in identifying the second of two targets when the second is presented temporally close to the first (attentional blink or AB; Raymond, Shapiro, \& Arnell, 1992). A similar phenomenon is the slowing of responses to the second of two targets if they are close in time ( psychological refractory period or PRP; Pashler \& Johnston, 1998). All of these phenomena indicate that during episodes in which cognitive processes are engaged in certain activities, capacity limits create difficulties in performing other activities or the same activity with other stimuli. When these capacity limits are exceeded by a high rate of information presentation (as in conventional tasks that use rapid serial visual presenta- tion, or RSVP) the above mentioned phenomena (i.e., RB, $\mathrm{AB}$, and PRP) show up, all of which reveal different types of performance decrements.

A similar phenomenon, although much less studied, is the report of a mixture of features between stimuli presented in close temporal succession, sometimes called intrusions or illusory conjunctions in the time domain (Botella, Barriopedro, \& Suero, 2001; Botella \& Eriksen, 1992; Cohen \& Ivry, 1989; Gathercole \& Broadbent, 1984; Keele, Cohen, Ivry, Liotti, \& Yee, 1988; Kikuchi, 1996; Lawrence, 1971; McLean, Broadbent, \& Broadbent, 1983). We identify stimuli by building integrated percepts or bound representations of significant units that give sense to the world around us. When presentation rates are within some "natural" range, the extracted features can be properly bound into representations of the presented objects. However, when the presentation rate exceeds a certain limit, incorrect combinations of the presented features can result. The study of the factors affecting the number and types of these errors helps us to understand how our cognitive system works. Different combinations

J. Botella, juan.botella@uam.es 
of a key dimension (the one that defines the target) and a response dimension (the one to be reported), with different presentation rates, generate different levels of performance and different patterns of intrusions in predictable ways - that is, with a predominance of pre- or posttarget intrusions or a balance between both types of errors (e.g., Botella, García, \& Barriopedro, 1992).

The term "illusory conjunction" was first used to refer to feature migrations between stimuli presented simultaneously but in different positions in the visual field (Treisman \& Gelade, 1980; Treisman \& Schmidt, 1982). Although illusory conjunctions in the space domain have been replicated in many studies, their very existence has been challenged by Donk (1999). When attentional capacity limits are exceeded, some of the features were originally described as free floating, and it was implicitly assumed that they could be more or less randomly recombined. Donk's (1999) position is a methodological criticism of the results alleged to support the hypothesis of random combination of free-floating features. She claimed that in many experiments, the misidentification of the displayed features (i.e., confusions in tagging identities to displayed items) is not differentiated, as a source of errors, from genuine illusory conjunctions. When misidentifications are taken into account, the number of errors is not larger than would be expected from totaling the sources of errors identified plus pure guesses.

However, it is not necessarily true (and is not essential for the feature integration theory) that the isolated features are recombined at random. Specifically, several experiments have shown that isolated features convey location information that can be used by the system to choose among them (Ashby, Prinzmetal, Ivry, \& Maddox, 1996; Cohen \& Ivry, 1989; Hazeltine, Prinzmetal, \& Elliott, 1997). Therefore, the recombination is not the result of "pure guessing," but rather "sophisticated guessing" based on imprecise location information. Although the debate is not closed (see Donk, 2001; Prinzmetal, Diedrichsen, \& Ivry, 2001), the evidence available strongly supports the idea that illusory conjunctions in the space domain are real, although the isolated features are not combined at random; rather, some attentional and spatial constraints modulate the probability of binding two specific features (see Quinlan, 2003).

In a similar vein, Botella et al. (2001) proposed a model of the formation of illusory conjunctions in the time domain, in which the choice of response features belonging to the target is not random, but sometimes results from sophisticated guessing. If the target features have not been properly bound, such guessing is distributed over the response features that are available at the moment when the target is detected. Thus, their proposal also contrasts with Donk's (1999) explanation of illusory conjunctions as methodological artifacts. ${ }^{1}$ Specifically, Botella et al. (2001) proposed a model in which responses are generated in two different ways in RSVP tasks. Assuming that the time needed to build an integrated percept can be thought of as a random variable, then, at the rates employed in RSVP experiments, the process of focal attention will succeed in integrating the target features on a specific propor- tion of the trials and will essentially generate hits. However, on trials in which focal attention does not succeed in target integration before it is interrupted by the arrival of new items, the observers have another opportunity for giving a correct response. This "plan B" consists of an attempt to exploit any featural information that is available in the system to make a guess from among the features of the response dimension that remain isolated in visual memory but have not been bound into a percept. Although the second attempt can produce a correct response, it is also the means by which errors of migration over time are produced. The distribution of errors around the target reflects the process by which such errors are generated.

As an extension of Botella et al.'s (2001) model, it is proposed here that the choice of which response features to report when focal attention fails to bind the target features can be described by an application of the constant ratio rule derived from Luce's (1959; see Logan, 2004) choice axiom. The constant ratio rule is assumed to be applied over the levels of activation of the representations of response features of stimuli presented around the target. We will not repeat here the arguments that support the original description of the model (see Botella et al., 2001). Rather, in the present article, we focus on predictions derived from the model for the distribution of errors around the target, and present new empirical evidence using a double response paradigm.

More importantly, the results with the double response paradigm will help us test a core idea of parallel models, for which the evidence is scarce. This core idea is that the features pertaining to the "to-be-reported" dimension are extracted in parallel, from several stimuli. The variability of the observed responses and the fact that they are distributed around the target have been taken to support that point. However, it is still possible that only one feature is extracted, and the distribution of errors reflects the probability that the only feature extracted is one selected from one of the positions around the target. Using the double response paradigm, we will test this idea by asking observers to report two response features instead of only one. We will determine whether the observers are able to report the second feature in a nonrandom way.

\section{Testing the Predicted Distribution With a Two-Response Paradigm}

The experimental paradigm we used consists of a conventional RSVP search task, but we required a second response in which the observer was to indicate his or her "second candidate." The idea of asking for several ordered answers is not new, since in a somewhat different context, it was fruitfully used by Reeves and Sperling (1986). They presented two lists of items in RSVP, one on each side of a fixation point. The observers had to report the identity of the item presented simultaneously with a target in the other stream, and also a few following items. The frequency of reporting the item from each position following the target reflected the "attentional window" employed. The results showed that the system processed and retained several items, although they were seldom reported in the exact order in which they had been shown. Rather, there 
appeared to be a distribution of memory strength for the items centered around a specific time delay after the cue occurred in the opposite stream.

In the present experiments, the task is not to report the target stimulus as such, but some isolated feature associated with the target item. This is an important difference from Reeves and Sperling's (1986) procedure, because they asked for a report of the actual stimuli presented, whereas we ask only for the response feature. We expect that the procedure is also fruitful when the observers are asked to report isolated features, as a way to study how the system manages the free-floating features described in feature integration theory (Treisman \& Gelade, 1980). Suppose we ask for the letter in red. If the representations available are "integral," and include the shape and the color, then the observers could easily discard them as candidates, because only one contains the "target-defining feature." In Reeves and Sperling's work, there was no possible confusion, because all of the items were presented in the same color, but in our experiments, those confusions are the main effect under study.

We will check first on the ability of the observers to perform the task. If they are able to make a second choice, and this choice is not randomly selected from the set of response alternatives, then the system must have more than one response feature available that was likely to have been part of the target. This result would reinforce the idea that the stimuli are processed in parallel, and that at least some of the features from the response dimension are extracted and maintained simultaneously (see Keele \& Neill, 1978). This result would also oppose a point of view (one of the varieties of early selection) that only the features of the selected item are extracted, whether correct or not (e.g., Broadbent, 1977; Lawrence, 1971; McLean et al., 1983).

Second, we will study the occurrence distribution of the second responses. Especially important will be the relationship between both responses. From Botella et al.'s (2001) model, it is predicted that the second response will not be independent of the first one. Specifically, the conditional probability of choosing the response feature from a specific position around the target, given the response selected in the first response, will depend on the serial position of the feature reported as the first response.

\section{Some Quantitative Predictions}

In Botella et al.'s (2001) model, the trials that yield intrusion errors are trials resulting from a decision made under uncertainty, taking as a basis the residual visual information. "Sophisticated guessing" in this case consists of choosing among the alternatives with a probabilistic mechanism based on the application of Luce's axiom to the levels of activation of the response features. The constant ratio rule derived from that axiom predicts, as a consequence, that the relationship between the percentages of choices should reflect that application, both in the first and second responses. As has been mentioned, this is only applied to the errors and to that part of the hits produced as "fortunate conjunctions," once focalization has failedthat is, all responses generated by the sophisticated guessing mechanism. To do that, we first need an estimation of the hits produced by that mechanism. The results of previous experiments show that a quadratic function fits the data reasonably well (e.g., those from simulations by Botella et al., 2001). We will use that function to estimate the percentage of responses that are hits produced by the sophisticated guessing mechanism.

Let us see how Luce's (1959) axiom can be applied to the percentages of choices of each alternative on each trial. The basic idea is contained in the constant ratio principle. Let us suppose that in a specific experimental condition, the percentages of choices (generated by the sophisticated guessing mechanism) for the five alternatives $(-2,-1,0$, +1 , and +2 , with 0 being the position of the target in the RSVP sequence) in the first response are 5, 10, 25, 20, and 5 (the other $35 \%$ are hits produced by the focalization mechanism). If the first and second responses are based on the same information and are generated by applying the same procedure, then the relationship between the choices for each pair of alternatives observed in the first response must reappear in the second response. Thus, between the alternatives -2 and -1 , there is a relationship of $1: 2$, whereas the relationship between the -2 and +2 items is $1: 1$. Let us focus, for example, on the trials in which the observer chooses as the first response the feature from the +1 position. The ratio between the selections of the -2 and -1 features in the second response and between the $(-2)$ and $(+2)$ items, must still be $1: 2$ and $1: 1$. For studying the fit of this prediction, we will calculate first the prediction for each alternative in the second response, conditionalized on the response given in the first one. It is important to highlight that for making these predictions, we will use only the frequencies of choices observed in the first responses. Then we will calculate the ratios between the empirical frequencies of choices for each cell in the second responses. In short, we will have on one side, the predictions for the ratios of the conditional proportions for the second responses, based exclusively on the first responses; on the other side, we will have the same ratios, but calculated over the empirical proportions in the second responses. We will calculate the correlation between both sets of quantities and interpret the magnitude as an assessment of the predictive capacity of the first responses for the second responses and, thus, of the predictive capacity of the model's assumptions.

Let us go back to the previous numerical example, in which the first response corresponds to the feature from position +1 . According to the data from the first responses, given that the observer has available only the features from positions $-2,-1,0$, and +2 for making a second response, these features should be chosen in the following proportions: (1) for the -2 item, $5 /(5+10+25+5)$; (2) for the -1 item, $10 /(5+10+25+5)$; (3) for the 0 item, $25 /(5+$ $10+25+5)$; (4) for the +2 item, $5 /(5+10+25+5)$. These predictions are based exclusively on the first responses. Taking now the trials in which the feature from position +1 is selected for the first response, we can calculate the empirical ratios of frequencies of second responses that parallel those four ratios. We will apply this procedure to all cells of the contingency table, with the exception of the main diagonal, which will be empty because the ob- 
servers were not allowed to make the same choice in both responses.

\section{The Present Study}

In the present experiments, we used a two-response paradigm. In order to estimate the conditional probabilities between both responses, we needed a larger number of trials than is usual when only one response is required. Therefore, each observer participated in 10 sessions of 250 trials each. In Experiment 1, the key dimension was the identity of the letter, and the response dimension was its color (e.g., "In what color does 'T' appear?"), whereas in Experiment 2, the roles were interchanged (e.g., "Which letter appears in red?"). In both cases, the participants were asked to give a second response with their "second candidate" - that is, they were to identify the feature that they would have reported if the one selected in the first choice was not available.

\section{EXPERIMENT 1}

\section{Method}

Participants. Five students with normal or corrected-to-normal vision from the Department of Psychology at the Autónoma University of Madrid served as voluntary participants.

Stimuli and Apparatus. The experiment was controlled by an IBM-compatible PC. The experimental program was written and run using Micro Experimental Laboratory (MEL; Schneider, 1988).

We prepared a total of 250 series of 13 letters each. Each series was composed of 13 different uppercase letters. The critical set (defined as the target plus the two items before it and after it) could occupy three different positions in the series $(4-8,5-9$, or $6-10)$, in order to reduce the predictability of when the target could occur and to separate the critical set from the beginning and the end of the series by several items. The letters were presented in five different colors (red, yellow, blue, green, and white) against a background that remained gray throughout the experiment. The five colors were employed for the five stimuli from the critical set, randomly assigned to each series. For the letters of each series outside the critical set, the four colors not used for the target letter were randomly assigned, with the only restriction being that two consecutive stimuli were never the same color.

Procedure. Each of the 10 sessions consisted of 250 trials. The participant sat at a $40-\mathrm{cm}$ viewing distance from the screen, from which the letters of the series had a visual angle of $0.46^{\circ}$ in height and $0.29^{\circ}$ in width.

At the beginning of each trial, the letter target for that trial appeared in the center of the screen, in black. The participant began the series by pressing the space bar. Each letter remained on the screen for $83 \mathrm{msec}$, being replaced immediately by the next one, and so on until the end of the series. Then a response menu was displayed on the screen. The menu always included the names of the same five colors, numbered 1 to 5 (each in its own color) and in the same order, plus "don't know" as the sixth option, in black.

Table 1

Distribution of the Mean Percentages of the First Responses Within the Critical Set in Experiments 1 and 2

\begin{tabular}{cccccc}
\hline & \multicolumn{5}{c}{ Response } \\
\cline { 2 - 6 } Experiment & -2 & -1 & Hit & +1 & +2 \\
\hline 1 & 6.0 & 9.4 & 56.2 & 22.7 & 5.6 \\
2 & 5.3 & 14.2 & 52.4 & 23.9 & 4.2 \\
\hline
\end{tabular}

The participant then had to make a response by pressing a key, from 1 to 6 on the keyboard, specifying in which color the target had appeared. Then the response menu was presented again, prompting an answer about the second candidate; the response procedure was the same as for the first one (pressing a key from 1 to 6). Each session included 18 practice trials before the experimental block; we excluded practice trials from the data analysis.

\section{Results}

We will present the results in two sections: first, the results of the first responses, and second, those of the second responses and their relationships with the first responses.

First responses analysis. We obtained the distribution of responses for each participant. The mean percentage of "don't know" responses was 6\%. For the rest of the trials, we calculated for each participant the percentage of responses in which the reported feature belonged to the stimulus in each position in the critical set. The first row of Table 1 shows the means of these percentages. Although the mean percentage of hits is $56.2 \%$, the distribution of intrusions shows a clear predominance of posttarget errors $(28.3 \%)$, in comparison with the pretarget errors (15.4\%). These results are consistent with those reported in previous similar experiments in which only one response was required (e.g., Botella, 1992; Botella \& Eriksen, 1991, 1992; McLean et al., 1983).

As described in the introduction, we estimated the number of responses that are hits produced by the sophisticated guessing mechanism by fitting a quadratic function to the figures in the $-2,-1,+1$, and +2 cells. $^{2}$ The fitted function accounts for $62.3 \%$ of the variance and allows an estimation of $19.5 \%$. That is, the $56.2 \%$ hit rate can be decomposed into $36.7 \%$ from focalization and $19.5 \%$ from "fortunate conjunctions" resulting from the sophisticated guessing mechanism. This value will be employed in the next section.

Second responses analysis. In order to analyze the second responses and their relationships with the first responses, we selected the trials for which neither of the responses were "don't know." This option was selected as the second response in $11.7 \%$ of the trials, on average. When the first response was "don't know," the second one was practically always “don't know” as well (99.9\%).

For the trials in which the two responses contained features from the stimuli of the critical set, we calculated the proportions of choices for each alternative in the second response, conditionalized on the choice of each one in the first response. Table 2 shows the average of the 5 observers in each cell after calculating the values for each participant.

It is very clear from the data in Table 2 that the distributions of second responses vary as a function of the first response. We have analyzed each conditional distribution with a one-way, within-subjects ANOVA. In all five rows, there is a significant effect of position, indicating that the "all cells equal" hypothesis can be rejected for each conditional distribution $\left[F_{\mathrm{s}}(3,12)=3.78,9.23,5.97,8.23\right.$, and 10.92, respectively; $p \mathrm{~s}<.05]$.

In order to analyze these distributions under Botella et al.'s (2001) model, we have derived predictions using 
Table 2

Mean Percentages of Responses in Experiment 1 Corresponding to Each Option of the Critical Set in the Second Response, Conditionalized on the Option Chosen in the First Response

\begin{tabular}{|c|c|c|c|c|c|c|}
\hline & & \multicolumn{5}{|c|}{ Response 2} \\
\hline & & -2 & -1 & 0 & 1 & 2 \\
\hline \multirow{5}{*}{ Response 1} & -2 & & $\begin{array}{l}23.6 \\
(16.4 ; 21.0)\end{array}$ & $\begin{array}{l}31.0 \\
(34.9 ; 29.2)\end{array}$ & $\begin{array}{l}26.0 \\
(39.7 ; 31.8)\end{array}$ & $\begin{array}{l}19.5 \\
(9.8 ; 18.0)\end{array}$ \\
\hline & -1 & 20.1 & & 35.3 & 29.3 & 15.3 \\
\hline & 0 & $\begin{array}{l}19.4 \\
(13.7 ; 19.8)\end{array}$ & $\begin{array}{l}28.7 \\
(21.5 ; 23.4)\end{array}$ & & $\begin{array}{l}34.4 \\
(51.9 ; 37.4)\end{array}$ & $\begin{array}{l}17.5 \\
(12.8 ; 19.4)\end{array}$ \\
\hline & +1 & $\begin{array}{l}16.7 \\
(14.8 ; 20.3)\end{array}$ & $\begin{array}{l}22.3 \\
(23.2 ; 24.2)\end{array}$ & $\begin{array}{l}38.9 \\
(48.1 ; 35.7)\end{array}$ & & $\begin{array}{l}22.1 \\
(13.8 ; 19.8)\end{array}$ \\
\hline & +2 & $\begin{array}{l}18.5 \\
(10.4 ; 18.3)\end{array}$ & $\begin{array}{l}17.9 \\
(16.3 ; 21.0)\end{array}$ & $\begin{array}{l}26.3 \\
(33.9 ; 29.1)\end{array}$ & $\begin{array}{l}37.3 \\
(39.4 ; 31.7)\end{array}$ & \\
\hline
\end{tabular}

Note-The numbers in parentheses show first the predicted values following Luce's axiom assumed in the model (see the introduction section) and, second, the predicted values adding a number of random responses in the second response (see the Discussion of Experiment 2).

the procedure described in the introduction. Those predictions are exclusively based on the distribution of frequencies of the first responses, as they appear in the first row of Table 1, plus the value estimated for hits from fortunate conjunctions (19.5; also estimated from first responses). The predictions refer to the 20 cells outside the main diagonal. We calculated the correlation between these predictions and the corresponding empirical values as obtained in the second responses. The correlation is $r=.887(p<$ .01 ). Table 2 shows (as the first value within the parentheses) the predicted conditionalized probabilities for the 20 cells.

\section{Discussion}

The first goal of Experiment 1 was to test whether observers were able to report a "second candidate" for the response feature of the target on the basis of such featural knowledge retained in memory or on the basis of random guesses. The results show clearly that the second response is not selected at random by the observers. Rather, the claim that several response features are extracted and maintained long enough to form the basis of sophisticated guessing is empirically supported. The choice probabilities likely reflect the relative levels of activation of feature representations, and those levels of activation are probably closely linked to their positions in the series, in relation to the target.

From Botella et al.'s (2001) model, it is proposed that on trials in which the target's features are not bound by focal attention, they remain floating in the system as partial representations of features from the response dimension with different levels of activation. These features can be employed by the system as the basis for guesses. The second goal of the present experiment was to test the model's derived claim that the application of the constant ratio rule to those levels of activation estimates the distribution of responses in the menu shown on each trial. With the data from the second responses, we have tested the prediction derived from this proposal. The correlations obtained have shown that the basis for both responses is effectively the same. The second responses keep a certain global relationship with the response given first. Thus, when performing tasks of perceptual integration with RSVP, the observers apparently extract and maintain the features from several stimuli in parallel.

In order to increase the generality of the conclusions, we completed a second experiment, with the relevant dimensions interchanged. It could be argued that the good fit obtained was based on the small number of observers or perhaps that it is a phenomenon that is specific for the task of reporting the color of the target letter. In order to address these issues, we repeated the experiment, with the same series of stimuli, but now with the task of identifying the letter presented in a specified target color. That is, we interchanged the roles of the relevant dimensions, so that now the key dimension is color and the response dimension is the identity of the letter in the target color.

Furthermore, in Experiment 1 it is possible (although perhaps unlikely), that the observers employed the strategy of looking for the color that was unique in the series, because the color of the target letter was used only for that letter within each trial. In Experiment 2 they cannot employ that strategy, because the to-be-reported feature (the identity of the letters) was not repeated within a trial. A similar pattern of results in Experiment 2 should minimize the effects of the confounding in Experiment 1.

\section{EXPERIMENT 2}

\section{Method}

Participants. Five different participants were chosen from the same pool as in the previous experiment.

Stimuli and Apparatus. The stimuli and apparatus were the same as those used in Experiment 1.

Procedure. The procedure was the same as in Experiment 1, with two exceptions: First, the information for defining the target in each trial was given by showing " $\& \& \&$ " in the target-defining color for that trial. Second, the response menu included the five letters of the critical set - numbered, in black, and in a random order - plus the option "don’t know."

\section{Results and Discussion}

First response analysis. In $1.4 \%$ of trials, on average, the observers' response was “don't know.” The second row 
of Table 1 presents the observed response distribution for the rest of the trials. As in the previous experiment, the results indicate that both the average percentage of hits $(52.4 \%)$ and the pattern of intrusions $(28.1 \%$ post and $19.5 \%$ pre) converge with those of previous experiments in the field. Here, the distribution between pre- and posttarget errors is more balanced than in Experiment 1.

The quadratic function, fitted as in Experiment 1, accounts for $83.3 \%$ of the variance, and the value estimated for hits is 23.8 . That is, the $52.4 \%$ hit rate can be decomposed into $28.6 \%$ of hits by focalization and $23.8 \%$ of "fortunate conjunctions" produced by the sophisticated guessing mechanism.

Second responses analysis. The option "don't know" was chosen as the second response in $10.2 \%$ of the trials, on average. When the first response was "don't know," the second one was also "don't know," in 99\% of the trials.

The ANOVAs for the five conditional distributions again all yielded significant effects, indicating that the empirical distributions deviate from the uniform (pure guessing) hypotheses $\left[F_{\mathrm{S}}(3,12)=5.77,14.32,6.76,11.21\right.$, and 9.18 , respectively; $p s<.05]$.

Employing the same procedures as in the previous experiment, we prepared Table 3 , in which the mean response percentages are shown. Using the same procedure as in the previous experiment, we obtained the correlation between the predictions based on the application of the constant ratio rule to the first responses and the observed frequencies in the second response. The correlation between the averages is $r=.956(p<.01)$. This indicates, as in the previous experiment, that both responses are probably selected on the same basis. Table 3 shows again as the first value within the parentheses the predicted conditionalized probabilities for the 20 cells.

A close inspection of Tables 2 and 3 shows some regularity in the differences between obtained and predicted values. The empirical values are lower than the predicted values in the central columns (hits) and in most neighbor cells (positions \pm 1 ) - that is, in those cells where the predicted values are high. On the other hand, in the cells far from the center (positions \pm 2 ) - just those where the predicted values are small - the empirical values exceed the predictions. This regularity deserves some attention.

It appears as though the observed trend in the deviations between predicted and empirical values is due to some type of regression toward the mean in the actual data. This approach to the "average" is what would be observed if on some proportion of the trials the observers in fact make random responses. Suppose that this happens with probability $g$ after the first response has been given. Then, the theoretically predicted value for each cell $(T)$ should be corrected in a new one $\left(T^{\prime}\right)$ by $T^{\prime}=(1-g) T+(g / 4)$, because when a random response is given, the four alternatives available have the same probability of being selected for the response $(\mathrm{g} / 4)$. The least-squares estimate for parameter $g$ is .538 for Experiment 1 and .335 for Experiment 2.

The interpretation of parameter $g$ deserves some attention before we assess any resulting improvement in the model's fit. In many models for illusory conjunctions, it is recognized that some responses will be "random guesses." But many of them (and of course the one identified here) are not real guesses, in the sense that the observer makes a blind response (as if his or her eyes were closed during the trial). Naturally, an observer willing to be perceived as cooperative with the experimenter can avoid the "don't know" responses, making some random responses and being aware that those responses are pure guesses.

Suppose that in some proportion of the trials, the information related with the second response is lost or degraded while the observer is giving the first response. In the trials in which that happens, the second response will be selected from the disorganized and fragmented information still available from the stream of letters. Instead of being governed by a Luce's (1959) rule associated with the position in the series, any of the features presented in that trial are potentially selected with the same probability for the response. As a consequence, the responses look as if they were pure guesses, but in fact they are the product of a random mix of information from which the unaware observer gives what is considered to be a good response.

As a second example, suppose that in a trial, the observer is asked to report the letter in red, and that the se-

Table 3

\begin{tabular}{|c|c|c|c|c|c|c|}
\hline \multicolumn{7}{|c|}{$\begin{array}{c}\text { Mean Percentages of Responses in Experiment } 2 \text { Corresponding to Ea } \\
\text { Option of the Critical Set in the Second Response, Conditionalized } \\
\text { on the Option Chosen in the First Response }\end{array}$} \\
\hline & & \multicolumn{5}{|c|}{ Response 2} \\
\hline & & -2 & -1 & 0 & 1 & 2 \\
\hline \multirow{5}{*}{ Response 1} & -2 & & $\begin{array}{l}21.3 \\
(21.5 ; 22.7)\end{array}$ & $\begin{array}{l}33.1 \\
(36.0 ; 32.3)\end{array}$ & $\begin{array}{l}31.7 \\
(36.2 ; 32.4)\end{array}$ & $\begin{array}{l}13.9 \\
(6.4 ; 12.6)\end{array}$ \\
\hline & -1 & $\begin{array}{l}13.4 \\
(9.3 ; 14.5)\end{array}$ & & $\begin{array}{l}36.8 \\
(41.6 ; 36.0)\end{array}$ & $\begin{array}{l}37.3 \\
(41.8 ; 36.2)\end{array}$ & $\begin{array}{l}12.5 \\
(7.3 ; 13.3)\end{array}$ \\
\hline & 0 & $\begin{array}{l}15.9 \\
(11.1 ; 15.8)\end{array}$ & $\begin{array}{l}28.1 \\
(29.8 ; 28.2)\end{array}$ & & $\begin{array}{l}41.3 \\
(50.2 ; 41.8)\end{array}$ & $\begin{array}{l}14.8 \\
(8.8 ; 14.2)\end{array}$ \\
\hline & +1 & $\begin{array}{l}15.6 \\
(11.2: 15.8)\end{array}$ & $\begin{array}{l}22.1 \\
(29.9: 28.3)\end{array}$ & $\begin{array}{l}41.2 \\
(50.1: 41.7)\end{array}$ & & $\begin{array}{l}21.1 \\
(8.8: 14.3)\end{array}$ \\
\hline & +2 & $\begin{array}{l}11.3 \\
(7.9 ; 13.6)\end{array}$ & $\begin{array}{l}17.9 \\
(21.1 ; 22.4)\end{array}$ & $\begin{array}{l}30.7 \\
(35.4 ; 31.9)\end{array}$ & $\begin{array}{l}40.1 \\
(35.6 ; 32.0)\end{array}$ & \\
\hline
\end{tabular}

Note-The numbers in parentheses show first the predicted values following the Luce's axiom assumed in the model (see the introduction section) and, second, the predicted values adding a number of random responses in the second response (see the Discussion of Experiment 2). 
quence in the critical set is F, S, D, U, K. Suppose that the observer misidentifies the target letter (D) as some other one. Note that we are not saying that the letters are correctly identified and wrongly bound with other colors. We are saying that the shape " $\mathrm{D}$ " can be misidentified as the letter $\mathrm{O}$, for example, or any other curved letter. If in that trial the letter $\mathrm{O}$ is also presented in the critical set, then the response will be coded as an intrusion from the position where the letter $\mathrm{O}$ was presented. In short, a misidentification error is coded as a binding error of correctly identified features (as a genuine illusory conjunction).

The two sources described above illustrate how the probability of having a "random" response (the $g$ value) is influenced by the probabilities that various sources contribute to but are indistinguishable from random responses involved in any trial. Thus, in Experiment 1 (in which the color must be reported) there are only the same five colors in any trial. In Experiment 2 (in which the letter must be reported) there are five critical letters that change from trial to trial and are never repeated in the series, even outside the critical set. Given these differences, the very nature of the features involved, and the specific discriminability of the exemplars employed, there is no reason to expect that the parameter $g$ should be the same for any experiment with a task similar to the one employed here.

The predicted values with the model incorporating the parameter $g$ appear as the second value within the parentheses in Tables 2 and 3. It is obvious that these values are much closer to the empirical numbers than those predicted from the previous, simpler model. Given that the only difference between both models is that the second one incorporates the $g$ parameter (the second one is nested in the first one) it is appropriate to test whether the addition of another parameter significantly reduces the error variance (see, e.g., Maxwell \& Delaney, 1990). The result is statistically significant for both experiments $[F(1,18)=$ $90.51, p<.001$, for Experiment 1 , and $F(1,18)=48.38$, $p<.001$, for Experiment 2]. That is, in both sets of data, the errors of prediction are significantly reduced when including an estimate of the number of responses "randomly" generated in the second responses.

Naturally, we wonder whether a parameter $g$ would also be necessary for the first responses. However, our analyses do not suggest that this would improve the model's fit. Apparently, the processing required for the second response suffers from interference by the first response, but the opposite does not occur, most likely due to the sequential nature of the two responses, with additional delays occurring before the second responses.

\section{GENERAL DISCUSSION}

The reported results show the utility of asking for a second response candidate in RSVP tasks with two relevant dimensions. Whereas the distribution of the first responses is indistinguishable from those obtained in experiments with only one response, the analysis of the second responses sheds light on the functioning of the system, at least in relation to two theoretical issues. The first issue is that it is possible to make an educated (not random) sec- ond response. Although we already know that the system can retain several items from an RSVP stream, in previous experiments those items were the stimuli themselves instead of their isolated features (e.g., Reeves \& Sperling, 1986). Here we have shown that several free-floating features are available; they must be isolated features instead of integral stimuli (a package of features already bound), because if not, the observers would realize that those stimuli do not contain the target-defining feature. The pure guessing hypothesis applied to the second response would predict flat conditional distributions. However, we have found empirical distributions deviating from that significantly, suggesting that it is possible to make an educated (not random) second response.

Several studies have shown that the features reported when producing illusory conjunctions in the space domain are not randomly chosen (e.g., Ashby et al., 1996; Cohen \& Ivry, 1989; Hazeltine et al., 1997). The features from certain spatial positions are more likely than others to be chosen, thus indicating that although the binding process that generates a target percept has not been completed, the available features have certain partial information associated with them that makes them "better" or "worse" candidates for a response. This information serves to make guesses that certainly generate errors, but it yields better performance than would uneducated guesses. In short, the way to disconfirm the "pure guessing" hypothesis (Donk, 1999 ) is to show that the probability of reporting a given feature is linked to some characteristic of the stimuli important for the ongoing task.

The features of the two dimensions employed to construct the stimuli used in the present experiments were selected at random for each trial. As a consequence, the "pure guessing" assumption predicts a uniform distribution of responses around the target in an RSVP stream. However, there is still another possibility that retains the idea that only one feature is extracted on each trial. The idea is that the response features in the menu have different levels of likelihood for the observers, according to how close they are in the perceptual multidimensional space to the response feature taken as the first response. ${ }^{3}$ That is, suppose that the shape of the letter S is the only response feature extracted when an observer is asked to report the letter in red. If the menu also contains the letters FTZC, then the shape of the letter C will more probably be selected as the second candidate, because it is the only one with curved lines and it is more similar to the shape selected in the first place. Taking as a basis multiple aspects of the features, the observer can calculate a "similarity" value to make decisions. Fortunately, the experimental procedure employed here rules out this alternative explanation. The random process followed for constructing the series, and the order of the features in the response menu, would produce a flat distribution of responses around the target. In fact, an observer following this procedure would generate a distribution indistinguishable from one generated at random.

In short, the results from the first responses in our two experiments, and in many other published studies, show that the pure guessing assumption is not tenable for il- 
lusory conjunctions in the time domain. What is new in the present research is extending the rejection of the pure guessing hypothesis to the second response as well.

The results support the idea that while a person is viewing the RSVP stream when searching for a target, possible response features from several successive stimuli are extracted and retained in parallel, and remain floating in the memory system for some time. If a target feature is detected, but the associated response feature is not bound to it by the focalization process ("consolidated," in Chun \& Potter's, 1995, terms), then the free-floating features can be used as the basis for guessing. Further, if two responses are required, it appears that guesses are generated by the same consistent rule.

The second issue has to do with the rule that is used to generate guesses. Our results suggest that the procedures for generating both responses, when they are wrong, are probably the same. In fact, the preferences shown in the first response allow accurate predictions of those shown in the second responses.

The constant ratio rule derived from Luce's (1959) choice axiom makes good predictions for the second choice response probabilities. However, the demands of making two successive responses while retaining featural information from the RSVP stream probably result in some capacity limits on the accuracy of the rule application. In that case, the data for the second responses are more likely to be compromised by additional "pure guesses," with a uniform distribution across response candidates, than are the data for the first responses.

This concatenation of pure and sophisticated guesses raises the question of whether we can be sure that any illusory conjunctions have in fact occurred. When a letter or a color is wrongly reported as the target, we cannot tell whether it is the result of an illusory conjunction, a sophisticated guess, or a random guess. We can, however, use the data from first and second guesses to estimate the probabilities of successful feature binding as well as to evaluate the validity of the sophisticated guessing mechanism as an explanation of the regularity in both first and second guess responses. We cannot be sure, but even if they are not actual illusory conjunctions, it is still interesting to study what happens in this paradigm. With it, we can study the kind of information the observer has available for making a response. That information provides for the observer a more solid basis for perceptual inferences about the environment.

Even in "natural" conditions, far from the difficult conditions of an RSVP task, errors and illusory conjunctions undoubtedly occur. The process of perception always entails some processes of inference and interpretation. Illusory conjunctions are one extreme case of such errors, but as in any other perceptual construction, our cognitive system employs the available information to develop a representation of the world around us. It is certain that our perception is not determined by random combinations of available features. It uses spatial and temporal proximities to maximize the probabilities that features originating from the same object are the ones that are in fact most usually bound together. Our conclusion here reinforces the idea that in interpreting the environment, we not only have integral percepts but also isolated features that are extracted and maintained in parallel, which can be used to reconstruct representations of external visual reality.

\section{AUTHOR NOTE}

The present research received financial support from the Spanish Ministerio de Ciencia y Tecnología, Project BSO2003-08908, for J.B. and M.S., and from a research fellowship from The Netherlands Organization for Scientific Research (NOW) for J.F.J. We are grateful to Eric Ruthruff, Mark Nieuwenstein, and an anonymous reviewer for their helpful comments on earlier versions of this article. Correspondence concerning this article should be addressed to J. Botella, Facultad de Psicología, Universidad Autónoma de Madrid, Campus de Cantoblanco, c/ Ivan Pavlov, 6, 28049 Madrid, Spain (e-mail: juan.botella@uam.es).

\section{REFERENCES}

Ashby, F. G., Prinzmetal, W., Ivry, R. [B.], \& Maddox, W. T. (1996). A formal theory of feature binding in object perception. Psychological Review, 103, 165-192.

Botella, J. (1992). Target-specified and target-categorized conditions in RSVP tasks as reflected by detection time. Bulletin of the Psychonomic Society, 30, 197-200.

Botella, J., Barriopedro, M. I., \& Suero, M. (2001). A model of the formation of illusory conjunctions in the time domain. Journal of Experimental Psychology: Human Perception \& Performance, 27, $1452-1467$.

Botella, J., \& Eriksen, C. W. (1991). Pattern changes in rapid serial visual presentation tasks without strategic shifts. Bulletin of the Psychonomic Society, 29, 105-108.

Botella, J., \& EriKsen, C. W. (1992). Filtering versus parallel processing in RSVP tasks. Perception \& Psychophysics, 51, 334-343.

Botella, J., García, M. L., \& Barriopedro, M. [I.] (1992). Intrusion patterns in rapid serial visual presentation tasks with two response dimensions. Perception \& Psychophysics, 52, 547-552.

Broadbent, D. E. (1977). The hidden preattentive processes. American Psychologist, 32, 109-118.

Chun, M. M., \& PotTer, M. C. (1995). A two-stage model for multiple target detection in rapid serial visual presentation. Journal of Experimental Psychology: Human Perception \& Performance, 21, 109-127.

Cohen, A., \& IvrY, R. [B.] (1989). Illusory conjunctions inside and outside the focus of attention. Journal of Experimental Psychology: Human Perception \& Performance, 15, 650-663.

DonK, M. (1999). Illusory conjunctions are an illusion: The effects of target-nontarget similarity on conjunction and feature errors. Journal of Experimental Psychology: Human Perception \& Performance, 25, 1207-1233.

DonK, M. (2001). Illusory conjunctions die hard: A reply to Prinzmetal, Diedrichsen, and Ivry (2001). Journal of Experimental Psychology: Human Perception \& Performance, 27, 542-546.

Gathercole, S. E., \& Broadbent, D. E. (1984). Combining attributes in specified and categorized target search: Further evidence for strategic differences. Memory \& Cognition, 12, 329-337.

Hazeltine, R. E., Prinzmetal, W., \& Elliott, K. (1997). If it's not there, where is it? Locating illusory conjunctions. Journal of Experimental Psychology: Human Perception \& Performance, 23, 263-277.

KANWISHER, N. (1991). Repetition blindness and illusory conjunctions: Errors in binding visual types with visual tokens. Journal of Experimental Psychology: Human Perception \& Performance, 17, 404-421.

Keele, S. W., Cohen, A., Ivry, R. [B.], Liotti, M., \& Yee, P. (1988). Tests of a temporal theory of attentional binding. Journal of Experimental Psychology: Human Perception \& Performance, 14, 444-452.

Keele, S. W., \& Neill, W. T. (1978). Mechanisms of attention. In E. C. Carterette \& M. P. Friedman (Eds.), Handbook of perception, Vol. 9: Perceptual processing (pp. 3-47). New York: Academic Press.

KIKUCHI, T. (1996). Detection of kanji words in a rapid serial visual presentation task. Journal of Experimental Psychology: Human Perception \& Performance, 22, 332-341. 
LAWRENCE, D. H. (1971). Two studies of visual search for word targets with controlled rates of presentation. Perception \& Psychophysics, 10, 85-89.

Logan, G. D. (2004). Cumulative progress in formal theories of attention. Annual Review of Psychology, 55, 207-234.

LuCE, R. D. (1959). Individual choice behavior: A theoretical analysis. New York: Wiley.

Maxwell, S. E., \& Delaney, H. D. (1990). Designing experiments and analyzing data: A model comparison perspective. Belmont, CA: Wadsworth/Thomson Learning.

Mclean J. P., Broadbent, D. E., \& Broadbent, M. H. P. (1983). Combining attributes in rapid serial presentation tasks. Quarterly Journal of Experimental Psychology, 35A, 171-186.

Pashler, H., \& Johnston, J. C. (1998). Attentional limitations in dualtask performance. In H. Pashler (Ed.), Attention, pp. 155-189. Hove, U.K.: Psychology Press.

Prinzmetal, W., Diedrichsen, J., \& Ivry, R. B. (2001). Illusory conjunctions are alive and well: A reply to Donk (1999). Journal of Experimental Psychology: Human Perception \& Performance, 27, 538-541.

Quinlan, P. T. (2003). Visual feature integration theory: Past, present, and future. Psychological Bulletin, 129, 643-673.

Raymond, J. E., Shapiro, K. L., \& Arnell, K. M. (1992). Temporary suppression of visual processing in an RSVP task: An attentional blink? Journal of Experimental Psychology: Human Perception \& Performance, 18, 849-860.

Reeves, A., \& Sperling, G. (1986). Attention gating in short-term visual memory. Psychological Review, 93, 180-206.

SCHNEIDER, W. (1988). Micro Experimental Laboratory: An integrated system for IBM PC compatibles. Behavior Research Methods, Instruments, \& Computers, 20, 206-217.
Shapiro, K. (2001). The limits of attention: Temporal constraints in human information processing. New York: Oxford University Press.

Treisman, A. M., \& Gelade, G. (1980). A feature-integration theory of attention. Cognitive Psychology, 12, 97-136.

Treisman, A. [M.], \& Schmidt, H. (1982). Illusory conjunctions in the perception of objects. Cognitive Psychology, 14, 107-141.

\section{NOTES}

1. Although Donk's (1999) challenge was directed specifically to illusory conjunctions in the space domain, we refer to it here because for any proposal related to the generation of any kind of illusory conjunctions, the position that must be nullified by default is that they are generated at random, as "pure guesses," or as random misidentifications of the features displayed.

2. That is, the frequencies of the four positions that according to the model are always produced by the sophisticated guessing mechanism $(-2,-1,+1$, and +2$)$ in the first row of Table 1 are adjusted to a function of the form $y=a+b x+c x^{2}$. Then, it is solved for the $x=0$ value, giving the relative frequency of "fortunate conjunctions" (quadratic functions have shown reasonable fits to distributions of responses as do those generated by simulation of Luce's, 1959, rule; Botella et al., 2001). Of course, the value estimated for $x=0$ and the four empirical values of the other positions must be rescaled to add to $100 \%$ when interpreted as estimates of conditional probabilities (expressed in percentages) for analyzing the second responses and fitting the final model.

3. We thank Eric Ruthruff, who directly suggested this possibility when reviewing the manuscript.

(Manuscript received May 31, 2006;

revision accepted for publication May 10, 2007.) 